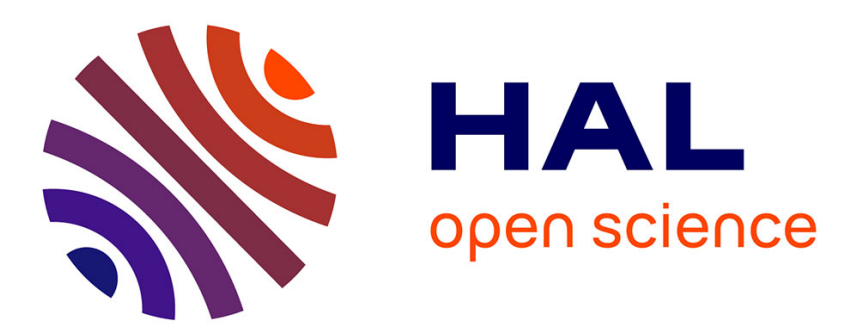

\title{
Is the Electromechanical Coupling the Driving Force for the Perpendicular Drift of First Class Cholesteric Finger?
}

\author{
L. Gil, S. Thiberge
}

\section{- To cite this version:}

L. Gil, S. Thiberge. Is the Electromechanical Coupling the Driving Force for the Perpendicular Drift of First Class Cholesteric Finger?. Journal de Physique II, 1997, 7 (10), pp.1499-1508. 10.1051/jp2:1997198 . jpa-00248528

\section{HAL Id: jpa-00248528 https://hal.science/jpa-00248528}

Submitted on 1 Jan 1997

HAL is a multi-disciplinary open access archive for the deposit and dissemination of scientific research documents, whether they are published or not. The documents may come from teaching and research institutions in France or abroad, or from public or private research centers.
L'archive ouverte pluridisciplinaire HAL, est destinée au dépôt et à la diffusion de documents scientifiques de niveau recherche, publiés ou non, émanant des établissements d'enseignement et de recherche français ou étrangers, des laboratoires publics ou privés. 


\title{
Is the Electromechanical Coupling the Driving Force for the Perpendicular Drift of First Class Cholesteric Finger?
}

\author{
L. Gil $\left(^{*}\right)$ and S. Thiberge \\ I.N.L.N. $\left({ }^{* *}\right)$, Université de Nice Sophia Antipolis, 1361 Route des Lucioles, \\ 06560 Valbonne. France
}

(Received 16 December 1996, revised 7 April 1997, accepted 6 June 1997)

\begin{abstract}
PACS.61.30.Gd - Orientational order of liquid crystals; electric and magnetic field effect PACS.11.30.Rd - Chiral symmetries
\end{abstract}

\begin{abstract}
When a cholesteric liquid crystal is sandwiched between two horizontal glass plates with homeotropic anchoring, chiral patterns named cholesteric fingers of first class, are known to occur. When a DC vertical electric field is applied, the Cholesteric Finger is then observed to move perpendicularly to its long-axis with a velocity $V_{\perp}$. In this paper, we prove analytically that this velocity may be related with the Lehmann rotation effect, and that it may provide a new way to measure the electromechanical coupling coefficient.
\end{abstract}

Résumé. - Dans un cristal liquide cholestérique pris en sandwich entre deux lames de verres conductrices avec des conditions d'ancrage homéotrope, l'apparition de structures spatiales isolées, appelées doigts cholestériques de première espèce, est maintenant bien comprise. Quand on soumet le cristal liquide à un champ électrique vertical. on observe alors que ces doigts cholestériques se mettent à bouger perpendiculairement à son grand axe avec une vitesse $V_{\perp}$. Dans ce papier, on montre analytiquement que cette vitesse pourrait être reliée à l'effet Lehmann, et que sa mesure permettrait alors d'estimer la valeur du coefficient de couplage électromécanique.

Chiral systems can sustain unusual cross couplings between hydrodynamic fluxes and forces [1]. A classical and spectacular illustration is the Lehmann rotation effect [2]. In 1900, Lehmann has observed that a cholesteric liquid crystal droplet, submitted to a thermal gradient parallel to its helical axis, displays a strong rotation of the local molecular axis. The effect has been discussed by Oseen in 1933 [3] and can be understood in the general framework of the hydrodynamic theory of cholesteric liquid crystal [4-7]. From the experimental point of view, the first measurements of the electromechanical coupling coefficient $\nu_{\mathrm{e}}$ (analogue to the thermomechanical effect, the role of the thermal gradient been played by the vertical DC electric field) has been performed in 1989 by Madhusudana and Pratibha [8]. They have observed that $\nu_{\mathrm{e}}$ is proportional to the wave vector of the helix, and have demonstrated its macroscopic origin $[9,10]$.

Cholesteric liquid crystals have also been the subject of many theoretical and experimental investigations of the unwinding transition. A typical experiment consists of a large-

$\left({ }^{*}\right)$ Author for correspondence (e-mail: gil@ms1.inln.cnrs.fr)

$\left({ }^{* *}\right)$ U.M.R. C.N.R.S. 129

(C) Les Éditions de Physique 1997 


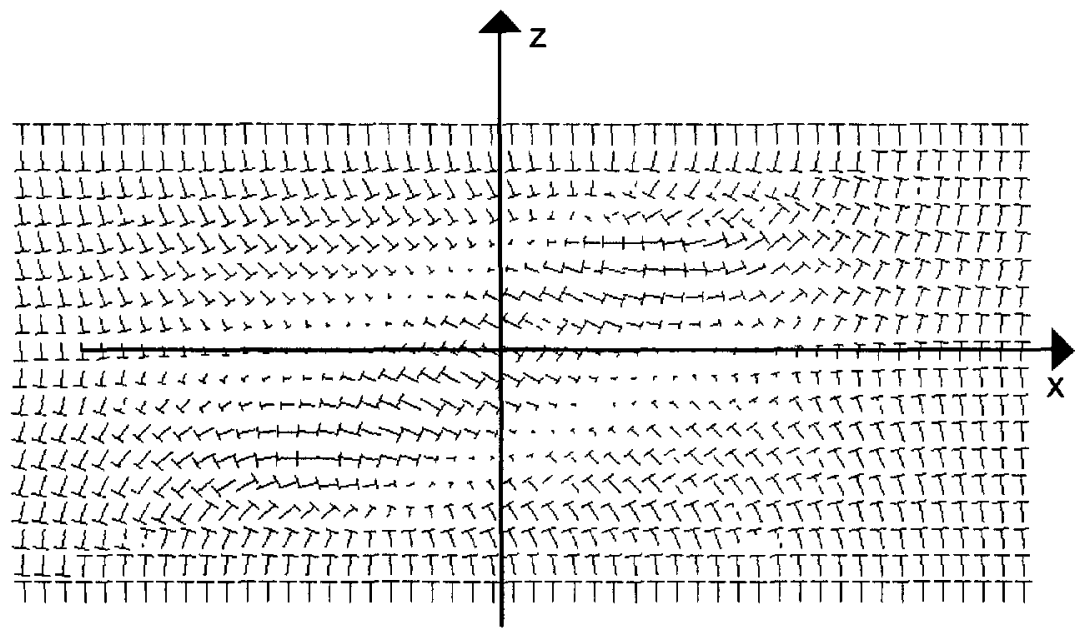

Fig. 1. - Usual nail representation of a thermodynamic equilibrium configuration of a first class cholesteric finger. The CF1 is assumed to be invariant with respect to translation along its long-axis (here the $y$ axis). The picture displays the transverse configuration ( $x$ and $z$ ). The numerical simulation is performed with a $400 \times 60$ mesh points, a space increment $\mathrm{d} l=0.2 \mu \mathrm{m}, K_{1}=5.3 \times 10^{-12} \mathrm{~kg} \mathrm{~m} \mathrm{~s}^{-2}$, $K_{2}=2.2 \times 10^{-12} \mathrm{~kg} \mathrm{~m} \mathrm{~s}^{-2}$ and $K_{3}=7.45 \times 10^{-12} \mathrm{~kg} \mathrm{~m} \mathrm{~s}^{-2}, \epsilon_{a}=-0.5, E=0.0$ volts m $\mathrm{m}^{-1}$, and $q_{0}=0.75 \times 10^{6} \mathrm{~m}^{-1}$. The picture is an enlargement of size $24 \mu \mathrm{m}$ by $12 \mu \mathrm{m}$ around the cholesteric finger. We have placed the origin of the $x$ and $z$ axis at the center of symmetry (see Eq. (7a)).

pitch cholesteric liquid crystal sandwiched between two horizontal parallel glass plates, with homeotropic anchoring. When the distance $d$ between the two plates is small compared to the cholesteric pitch $p$, the boundary conditions force all the molecules throughout the sample to be perpendicular to the plates. On the contrary, above a critical value of the confinement parameter $d / p$, cholesteric winding takes place. The thermodynamical phase diagram of the transition is very rich $[11,12]$, but we will deal here only with one single pattern, the Cholesteric Finger of the first class (CF1) [13], which is a long-shaped pattern, stretched between the two horizontal glass plates. Its width is about the sample thickness, its orientational order parameter configuration is continuous (for a numerical simulation see [14] and Fig. 1), and the two ends of the finger are not the same, one of them being sharper than the other. We focus onto the CF1, because it has recently been experimentally observed to move perpendicularly to its long-axis when submitted to a DC vertical electric field and then to give rise to the formation of archimedian spirals which rotate uniformly around their core [15-17].

In this paper, we want to theoretically establish the possible relationship between the electromechanical coupling and the perpendicular drift of the CF1 pattern. We compute an approximation of perpendicular velocity, show that it is proportional to the electromechanical coupling coefficient $\nu_{\mathrm{e}}$ and finally we check, in agreement with the experimental results [13], that the mean velocity has to vanish under the application of an $\mathrm{AC}$ vertical electric field.

In their pioneer paper, Hinov and Kukleva [15] have experimentally pointed out that the current-voltage characteristic of the cholesteric crystal under study is extremely important for the spiral formation. As usual in the study of the conductivity of dielectric liquids [18], they have observed the influence of the current-voltage prehistory of the cells, as well as the strong dependence on the treatment of the electrodes or on the impurity content, and they have concluded to the existence of a strong injection mechanism from the electrodes. Our 


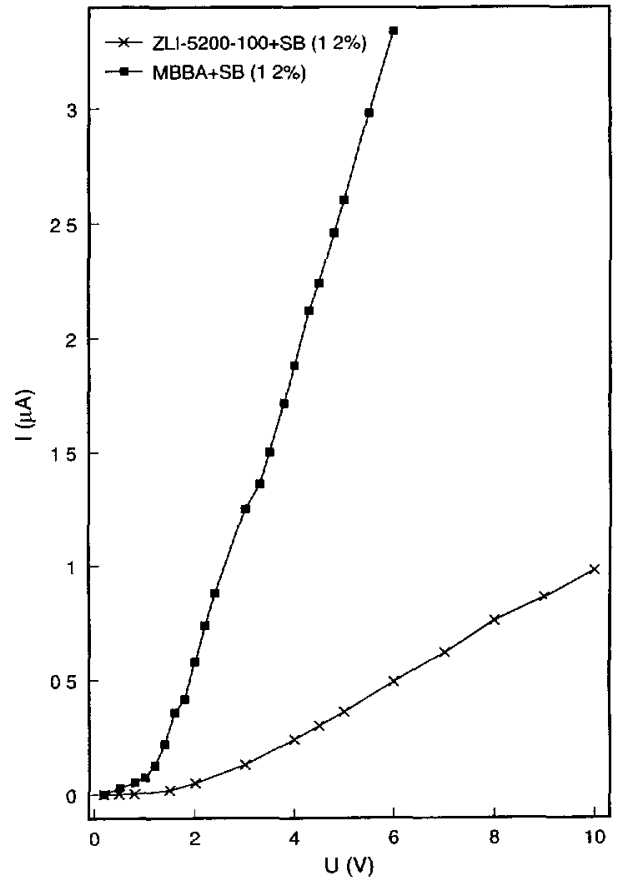

Fig. 2. - Current-voltage characteristic curves for MBBA/SB and ZLI5200-100/SB mixtures.

own observations are in agreement with their conclusions. For example, we have observed the formation of spirals in MBBA but not in a ZLI-5200-100 (Merck corp.) liquid crystals, both materials been doped $(1.2 \%)$ with a chiral side chain co-oligomer furnished by Wacker Chemie. This chiral dopant will be called SB in the following [19]. The temperature is the room temperature $\left(\simeq 23^{\circ}\right)$ and the distance $d$ between the two horizontal glass plates $d=12 \mu \mathrm{m}$. In the same time, we have measured the current-voltage characteristic of these two mixtures and have found that, inside the voltage range used, the electric intensity in the first mixture (MBBA/SB) is at least ten times higher than in the second one (ZLI/SB) (see Fig. 2). Therefore, the full description of the CF1 drift mechanism requires not only the general Ericksen-Leslie hydrodynamical theory of cholesteric liquid crystal [4-7], but also a description of the electric field $\mathbf{E}(\mathbf{r})$ through the sample (similarly the classical Lehmann effect requires a description of the heat flux). A priori, this electric field $\mathbf{E}$ (r) differs from the applied vertical electric field $\mathbf{E}_{0}$ because of the charge injection from the electrodes. Hence the full theoretical description is expressed as

$$
\begin{aligned}
& \left\{\begin{array}{l}
\rho\left(\frac{\partial V_{\beta}}{\partial t}+(\mathbf{V} \cdot \nabla) V_{\beta}\right)=\partial_{\alpha}\left(\sigma_{\alpha \beta}^{\mathrm{v}}+\sigma_{\alpha \beta}^{\mathrm{e}}+\mu_{1} n_{\alpha}(\mathbf{E} \wedge \mathbf{n})_{\beta}+\mu_{2} n_{\beta}(\mathbf{E} \wedge \mathbf{n})_{\alpha}\right) \\
F_{\beta}+\nu_{\mathrm{e}}(\mathbf{n} \wedge \mathbf{E})_{\beta}=\gamma_{1} N_{\beta}+\gamma_{2} n_{\alpha} A_{\alpha \beta} \quad \text { with } \quad A_{\alpha \beta}=\frac{1}{2}\left(\partial_{\alpha} V_{\beta}+\partial_{\beta} V_{\alpha}\right)
\end{array}\right.
\end{aligned}
$$

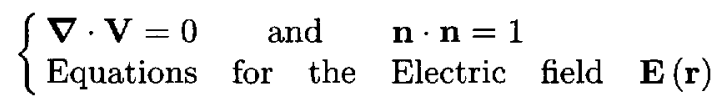

where $\rho$ is the mass density, $\mathbf{V}$ the velocity field, $\sigma_{\alpha \beta}^{\mathrm{v}}$ the viscous stress, $\gamma_{1}$ and $\gamma_{2}$ linear 
combinaison of viscous coefficients, $\sigma_{\alpha \beta}^{\mathrm{e}}$ the Ericksen stress. F the molecular field, is defined by

$$
F_{\alpha}=-\frac{\delta \mathcal{F}}{\delta n_{\alpha}}=-\left(\frac{\partial \mathcal{F}}{\partial n_{\alpha}}-\partial_{\gamma}\left(\frac{\partial \mathcal{F}}{\partial g_{\gamma \alpha}}\right)\right) \quad g_{\gamma \alpha}=\partial_{\gamma} n_{\alpha}
$$

where $\mathcal{F}$ is the Frank free energy density

$$
\begin{aligned}
\mathcal{F}= & \frac{K_{1}}{2}(\nabla \cdot \mathbf{n})^{2}+\frac{K_{2}}{2}\left(q_{0}+\mathbf{n} \cdot(\nabla \wedge \mathbf{n})\right)^{2} \\
& +\frac{K_{3}}{2}(\mathbf{n} \wedge(\nabla \wedge \mathbf{n}))^{2}-\frac{\epsilon_{0} \epsilon_{a}}{2}(\mathbf{n} \cdot \mathbf{E})^{2}
\end{aligned}
$$

$\mathbf{N}$ represents the rate of change of the director with respect to the background fluid and is expressed as

$$
\mathbf{N}=\frac{\partial \mathbf{n}}{\partial t}+(\mathbf{V} \cdot \nabla) \mathbf{n}-\omega \wedge \mathbf{n}
$$

where $\omega=\frac{1}{2} \nabla \wedge \mathbf{V}$ stands for the antisymmetric part of the gradient tensor $\partial_{\alpha} V_{\beta}$. Finally, $\mu_{1}$ $\mu_{2}$ and $\nu_{\mathrm{e}}$ are the three coefficients characterizing the electromechanical coupling.

Now some assumptions have to be done.

The first assumption deals with the hydrodynamic velocity. In 1900, Lehamnn has found that cholesteric liquid crystals spread between two glass surfaces, was set into motion by a flow of heat coming from below. He has observed that the different drops of liquid seemed to be rotating violently. but from optical studies, he concluded that it was not the drops themselves but the structure that was rotating. For the electromechanical coupling, the Madhusudana's observations [8] lead to the same conclusion. Finally, our own observations of the transverse drift of a CF1 are in agreement with these results. With the MBBA-SB mixture, electrohydrodynamical instabilities occur above a critical voltage of 3.5 volts, but we do observe spiral formation below this voltage, in a range between 2.2 and 3 volts. Therefore, it seems reasonable to assume a vanishing hydrodynamic velocity $\mathbf{V}$.

The second hypothesis deals with the electric charge repartition inside the sample. The equations describing the electric field involve physical and chemical processes as well (for a review, refer to [18]), and are so complex that we will even not try to write them. From an experimental point of view $[15,18]$, we know that a pronounced injection from the electrodes exists, confirmed by the existence of an inverse potential $\left(\mathbf{E}_{2}\right)$. Therefore, the simplest assumption we can make, consist then in assuming that $\mathbf{E}$ is constant through the sample, and just equal to the additional applied electric field

$$
\mathbf{E}=\mathbf{E}_{0}-\mathbf{E}_{2}
$$

Obviously this first order approximation is open to criticism, and the only justification is that it leads to the right qualitative behavior. It's worth noting that Madhusudana et al. [8] do not realize that they make the same kind of assumption (although they do observe a threshold voltage). Indeed, for their experimental measurements, they used the formula [8] (Eq. (11), p. 1830)

$$
\gamma_{1} \frac{\partial \phi}{\partial t}=K_{2} \frac{\partial^{2} \phi}{\partial z^{2}}-\nu_{\mathrm{e}} E
$$

where $z$ is the direction of the applied electric field, $\phi(z)$ is the angle between the director and the $z$ axis, and where $E$ is not the local electric field but $U / d$ with $U$ the difference of potential between the two plates. The confusion comes to the fact that in the usual thermomechanical 
case, the temperature is a linear function of $z$ [20], such that the gradient is constant through the sample. With these assumptions, equations (1) are then reduced to

$$
\left\{\begin{array}{l}
\gamma_{1} \frac{\partial \mathbf{n}}{\partial t}=\mathbf{F}(\mathbf{n})+\nu_{\mathrm{e}} \mathbf{n} \wedge \mathbf{E} \\
n^{2}=1
\end{array} \quad \text { with } \quad \mathbf{F}(\mathbf{n})=-\frac{\delta \mathcal{F}}{\delta \mathbf{n}}\right.
$$

Note that, when $\mathbf{E}=0$, the previous equation (2) is variationnal or potential [21] and just describes the fall down toward one minimum of the Frank distortion free energy. Therefore, the final asymptotic $(t \longrightarrow \infty)$ orientational configurations are necessarily stationary. Moreover, numerous experimental and theoretical results are available in this regime. Especially, the exact orientational configuration of the CF1 is numerically available [14,22]. This suggests to study equation (2) in a perturbative way, for small values of the electric field amplitude $(E=\epsilon \mathcal{E}$, $\epsilon$ being a small dimensionless parameter), and by means of a Melnikov-type analysis [23].

Because the CF1 is a long-shaped pattern, and because we are interested in the velocity of the perpendicular drift. we restrict ourselves to configurations invariant by translation along one direction (defined as the $y$ direction, and parallel to the CF1 long axis). Equation (2) can then be investigated through a $2 \mathrm{D}(x, z)$ analysis, $z$ being both the electric field and the vertical direction. We then look for a solution of the perturbated equation (2) with the following ansatz

$$
\mathbf{n}=\mathbf{n}^{0}\left(x+\xi\left(\epsilon t_{1}, \epsilon^{2} t_{2}, . .\right), z\right)+\epsilon \mathbf{n}^{1}+\epsilon^{2} \mathbf{n}^{2} .
$$

where $\mathbf{n}^{0}(x+\xi, z)$ corresponds to the orientational equilibrium configuration of a CF1 pattern, placed at $x=-\xi$ (Fig. 1). Equation (2) is invariant with respect to translation along the $x$ axis, but the presence of a CF1 breaks this continuous symmetry. As it costs almost no free energy to translate as a whole the CF1 along the $x$ axis [24], $\xi$ is then expected to slowly depend on time. The perpendicular velocity $V_{\perp}$ is given by

$$
V_{\perp}=\frac{\partial \xi}{\partial t}=\epsilon \frac{\partial \xi}{\partial t_{1}}+\epsilon^{2} \frac{\partial \xi}{\partial t_{2}}+. .
$$

After substitution in equation (2), we obtain at first order in $\epsilon$

$$
\left.D \mathbf{F}\right|_{\mathbf{n}^{0}}\left(\mathbf{n}^{1}\right)=\gamma_{1} \frac{\partial \mathbf{n}^{0}}{\partial x} \frac{\partial \xi}{\partial t_{1}}-\nu_{\mathrm{e}} \mathbf{n}^{0} \wedge \mathcal{E}
$$

and at second order

$$
\left.D \mathbf{F}\right|_{\mathbf{n}^{0}}\left(\mathbf{n}^{2}\right)=\gamma_{1}\left(\frac{\partial \mathbf{n}^{1}}{\partial t_{1}}+\frac{\partial \mathbf{n}^{0}}{\partial x} \frac{\partial \xi}{\partial t_{2}}\right)-\left.D^{2} \mathbf{F}\right|_{\mathbf{n}^{0}}\left(\mathbf{n}^{1}, \mathbf{n}^{1}\right)-\nu_{\mathrm{e}} \mathbf{n}^{1} \wedge \mathcal{E}
$$

where

$$
\begin{aligned}
& {\left[\left.D \mathbf{F}\right|_{\mathbf{n}^{0}}(\mathbf{V})\right]_{\imath}=\frac{\partial F_{2}}{\partial n_{\jmath}} V_{\jmath}} \\
& {\left[\left.D^{2} \mathbf{F}\right|_{\mathbf{n}^{0}}(\mathbf{U}, \mathbf{V})\right]_{\imath}=\frac{1}{2} \frac{\partial^{2} F_{2}}{\partial n_{\jmath} \partial n_{k}} U_{3} V_{k} .}
\end{aligned}
$$

Equations (3) and (4) are just linear equations in $\mathbf{n}^{1}$ and $\mathbf{n}^{2}$. They are of the form $\mathcal{L} n^{2}=\mathrm{rhs}^{\imath}$ with $i=1,2$, where $\mathcal{L}$ is the linear operator $\mathcal{L}=\left.D \mathbf{F}\right|_{\mathbf{n}^{0}}$, and where rhs stand for the right hand sides. Solutions can be found provided that the right hand side rhs ${ }^{2}$ belongs to the image of $\mathcal{L}$. These conditions, known as the solvability conditions, are not trivial because the Kernel 
of $\mathcal{L}$ is not vanishing since $\frac{\partial \mathbf{n}^{0}}{\partial x} \in \operatorname{Ker}(\mathcal{L})$. For our problem, it is easy but quite tedious to show that they are expressed as

$$
\begin{gathered}
\left\langle\frac{\partial \mathbf{n}^{0}}{\partial x}, \frac{\partial \mathbf{n}^{0}}{\partial x}\right\rangle \gamma_{1} \frac{\partial \xi}{\partial t_{1}}=\nu_{\mathrm{e}}\left\langle\mathbf{n}^{0} \wedge \mathcal{E}, \frac{\partial \mathbf{n}^{0}}{\partial x}\right\rangle \\
\left\langle\frac{\partial \mathbf{n}^{0}}{\partial x}, \frac{\partial \mathbf{n}^{0}}{\partial x}\right\rangle \gamma_{1} \frac{\partial \xi}{\partial t_{2}}=-\gamma_{1}\left\langle\frac{\partial \mathbf{n}^{1}}{\partial t_{1}}, \frac{\partial \mathbf{n}^{0}}{\partial x}\right\rangle+\left\langle\left. D^{2} \mathbf{F}\right|_{\mathbf{n}^{0}}\left(\mathbf{n}^{1}, \mathbf{n}^{1}\right), \frac{\partial \mathbf{n}^{0}}{\partial x}\right\rangle \\
+\nu_{\mathrm{e}}\left\langle\mathbf{n}^{1} \wedge \mathcal{E}, \frac{\partial \mathbf{n}^{0}}{\partial x}\right\rangle
\end{gathered}
$$

where $\langle$,$\rangle stands for the scalar product \langle\mathbf{A}, \mathbf{B}\rangle=\iint A_{2} B_{2} \mathrm{~d} x \mathrm{~d} z$. The interpretation of the first equation (5) is direct since $\mathbf{n}^{0}$ is already known (at least numerically) from the potential study. The resolution will give rise to a linear relation between the velocity and the electric field. On the contrary, it is much less easy to find a solution of the second solvability condition, since it requires the knowledge of $\mathbf{n}^{1}$. But as $\mathbf{n}^{1}$ depends on $E$, the resolution of equation (6) will give us some information about the way the velocity depends on $E^{2}$ and therefore the behavior of the CF1 under the application of an AC vertical electric field.

We are first considering equation (5). With our assumptions (equilibrium CF1 invariant by translation along $y$, and electric field parallel to $z$ ) the velocity is expressed as

$$
\frac{\partial \xi}{\partial t_{1}}=\frac{\nu_{\mathrm{e}} U}{\gamma_{1}} G
$$

where $U$ is the electric potential difference between the two plates $\left(E=\frac{U}{d}\right)$ and $G$ a dimensionless geometrical factor given by

$$
G=\frac{1}{d} \frac{\int_{-\infty}^{+\infty} \mathrm{d} x \int_{0}^{d}\left[n_{2}^{0} \frac{\partial n_{1}^{0}}{\partial x}-n_{1}^{0} \frac{\partial n_{2}^{0}}{\partial x}\right] \mathrm{d} z}{\int_{-\infty}^{+\infty} \mathrm{d} x \int_{0}^{d}\left[\frac{\partial n_{1}^{0}}{\partial x}+\frac{\partial n_{2}^{0}}{\partial x}+\frac{\partial n_{3}^{0}}{\partial x}\right] \mathrm{d} z}
$$

$G$ is a non vanishing coefficient. The exact dependence of $G$ on $U$ is investigated numerically (Fig. 3) in the case of a cholesteric liquid crystal with negative dielectric anisotropy $\left(\epsilon_{a}=-0.5\right)$. We have used a simple gradient method, with a projection of the gradient onto the constraint surfaces $n^{2}=1$. At each time step, the total free energy is computed and the new configuration is accepted only if the free energy is decreased. Spatial derivatives are computed by finite difference scheme (second order), on a grid of 200 times 30 collocations points, with a space increment $\mathrm{d} l=0.4 \mu \mathrm{m}$. Checking simulations have been performed with 400 times 60 points $(\mathrm{d} l=0.2 \mu \mathrm{m})$ and provide an error estimation. Finally we have used periodic boundary conditions in $x$, and rigid homeotropic conditions in $z$. Using for $\nu_{\mathrm{e}}$ the value obtained by Madhusudana [8] $\nu_{\mathrm{e}} \simeq 0.93 \times 10^{-6} \mathrm{C} \mathrm{m}^{-2}$, for $\gamma_{1}$ the typical MBBA value $\gamma_{1} \simeq 76.3 \times 10^{-3} \mathrm{~kg} \mathrm{~m}^{-1} \mathrm{~s}^{-1}$, and $U=0.5$ volts, we obtained for the perpendicular velocity $V_{\perp}$ the characteristic value $V_{\perp} \simeq 1 \mu \mathrm{ms}^{-1}$.

How can this result be compared with the experiment? In fact, we have not been able to experimentally measure the perpendicular drift of a straight isolated CF1. After the long time (several hours) application of a DC vertical electric field, the cholesteric sample is found to be filled with steadily rotating spirals. Obviously each spiral is slowed down by the neighbouring 


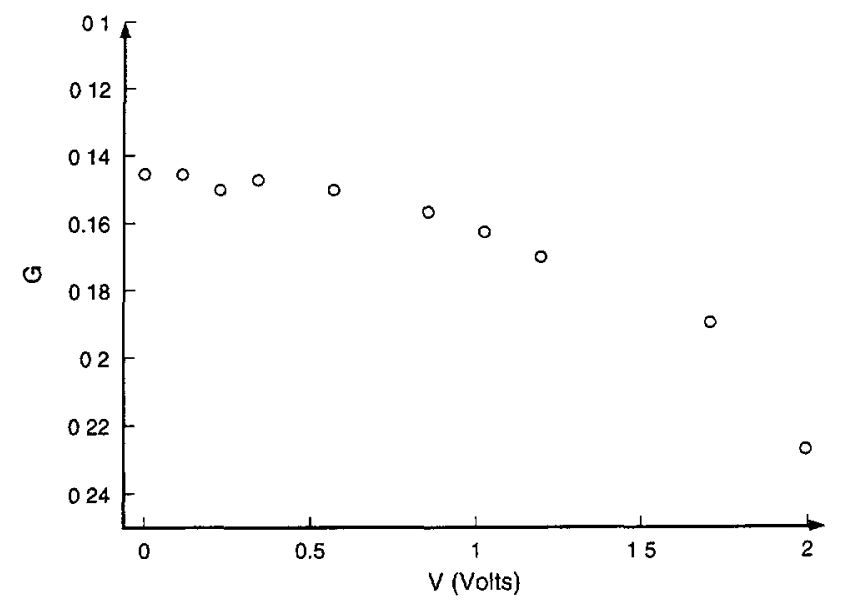

Fig. 3. - Evolution of the dimensionless geometrical factor $G$ versus $U$. We have used $200 \times 30$ mesh points with a space increment $\mathrm{d} l=0.4 \mu \mathrm{m}, K_{1}=5.3 \times 10^{-12} \mathrm{~kg} \mathrm{~m} \mathrm{~s}^{-2}, K_{2}=2.2 \times 10^{-12} \mathrm{~kg} \mathrm{~m} \mathrm{~s}^{-2}$, $K_{3}=7.45 \times 10^{-12} \mathrm{~kg} \mathrm{~m} \mathrm{~s}^{-2}$ and $\epsilon_{a}=-0.5$. Checking simulations with $400 \times 60$ mesh points provide an estimation of the relative error less than $3 \%$.

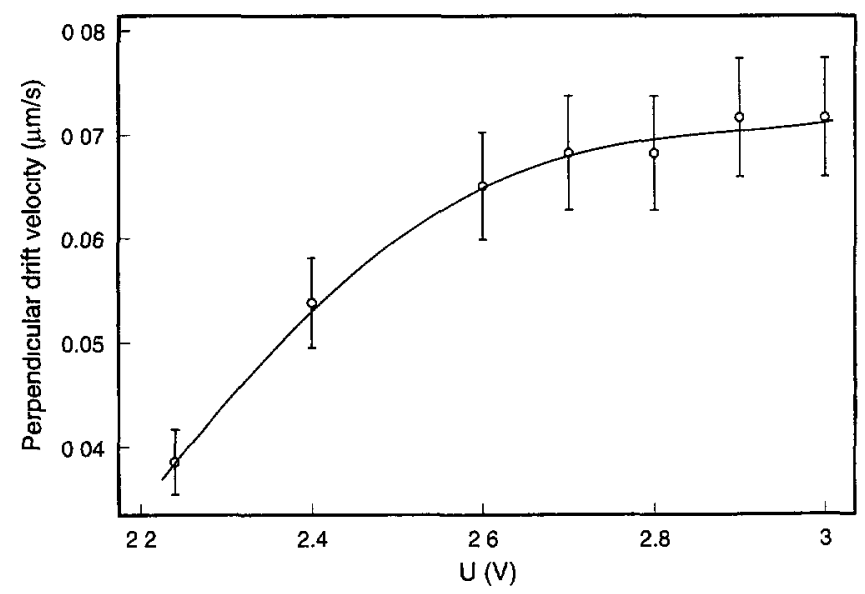

Fig. 4. - Experimental measurement of the perpendicular drift velocity of CF1 versus the applied tension $U$ between the two plates. The mixture used is MBBA/SB, the thickness is $12 \mu \mathrm{m}$. For each measurement, we have tried to select a steadily rotating, well formed double armed spiral.

spirals (accordingly our theoretical result is higher than the experimental measure) and we do not know how to estimate this breaking effect. However, in order to get an order of magnitude, we have then selected a single double armed spiral with at least 15 CF1 arms, have placed ourselves close to the sixth arm, and have measured the perpendicular velocity. The results are displayed in Figure 4. Taking into account, first the crudeness of the second assumption dealing with the homogeneity of the electric field through the sample, and second the experimental difficulty to isolate a single CF1 pattern, both theoretical and experimental results are not in too bad agreement. 
Now we are considering the equation (6), in order to characterize the CF1 behavior under an ac electric field. This is an important point because it has been experimentally used by Ribiere, Oswald and Pirkl [13] to establish the distinction between the cholesteric finger of first and second class, the former being at rest, the latter moving to give rise to spiral patterns (Ribiere [13], Gilli [25] and Sixou [26]). In this case, the injection mechanism can be neglected and the electric field $E$ inside the sample is just the applied electric field $E_{0}$.

The computation is based on symmetry arguments. Indeed, a careful analysis [27] of the CF1 equilibrium configuration $\mathbf{n}^{0}(x, z)$ (see Fig. 1) leads to the following observations

$$
\left\{\begin{array}{l}
n_{1}^{0}(-x,-z)=n_{1}^{0}(x, z) \\
n_{2}^{0}(-x,-z)=-n_{2}^{0}(x, z) \\
n_{3}^{0}(-x,-z)=n_{3}^{0}(x, z)
\end{array}\right.
$$

Therefore, the right hand side of equation (5) $\left(\mathrm{rhs}^{1}\right)$ obeys to

$$
\begin{cases}\operatorname{rhs}_{1}^{1}(-x,-z)=\left.\gamma_{1} \frac{\partial \xi}{\partial t_{1}} \frac{\partial n_{1}^{0}}{\partial x}\right|_{(-x,-z)}-\nu_{\mathrm{e}} \mathcal{E} n_{2}^{0}(-x,-z) & =-\operatorname{rhs}_{1}^{1}(x, z) \\ \operatorname{rhs}_{2}^{1}(-x,-z)=\left.\gamma_{1} \frac{\partial \xi}{\partial t_{1}} \frac{\partial n_{2}^{0}}{\partial x}\right|_{(-x,-z)}+\nu_{\mathrm{e}} \mathcal{E} n_{1}^{0}(-x,-z) & =+\operatorname{rhs}_{2}^{1}(x, z) \\ \operatorname{rhs}_{3}^{1}(-x,-z)=\left.\gamma_{1} \frac{\partial \xi}{\partial t_{1}} \frac{\partial n_{3}^{0}}{\partial x}\right|_{(-x,-z)} & =-\operatorname{rhs}_{3}^{1}(x, z)\end{cases}
$$

and the linear operator $\left.D \mathbf{F}\right|_{\mathbf{n}^{0}}$ to

$$
\left(\begin{array}{lll}
\frac{\partial F_{1}}{\partial n_{1}} & \frac{\partial F_{1}}{\partial n_{2}} & \frac{\partial F_{1}}{\partial n_{3}} \\
\frac{\partial F_{2}}{\partial n_{1}} & \frac{\partial F_{2}}{\partial n_{2}} & \frac{\partial F_{2}}{\partial n_{3}} \\
\frac{\partial F_{3}}{\partial n_{1}} & \frac{\partial F_{3}}{\partial n_{2}} & \frac{\partial F_{3}}{\partial n_{3}}
\end{array}\right)_{\mathbf{n}^{0}(-x,-z)}=\left(\begin{array}{rrr}
+\frac{\partial F_{1}}{\partial n_{1}} & -\frac{\partial F_{1}}{\partial n_{2}} & +\frac{\partial F_{1}}{\partial n_{3}} \\
-\frac{\partial F_{2}}{\partial n_{1}} & +\frac{\partial F_{2}}{\partial n_{2}} & -\frac{\partial F_{2}}{\partial n_{3}} \\
+\frac{\partial F_{3}}{\partial n_{1}} & -\frac{\partial F_{3}}{\partial n_{2}} & +\frac{\partial F_{3}}{\partial n_{3}}
\end{array}\right)_{\mathbf{n}^{0}(x, z)}
$$

These rules of transformation (7), together with equation (5), force $\mathbf{n}^{1}$ to satisfy

$$
\left\{\begin{array}{l}
n_{1}^{1}(-x,-z)=-n_{1}^{1}(x, z) \\
n_{2}^{1}(-x,-z)=+n_{2}^{1}(x, z) \\
n_{3}^{1}(-x,-z)=-n_{3}^{1}(x, z)
\end{array}\right.
$$

Finally, as $\left.D^{2} \mathbf{F}\right|_{\mathbf{n}^{0}}$ obeys to

$$
\left(\begin{array}{ccc}
\frac{\partial^{2} F_{1}}{\partial n_{1}{ }^{2}} & \frac{\partial^{2} F_{2}}{\partial n_{1}{ }^{2}} & \frac{\partial^{2} F_{3}}{\partial n_{1}{ }^{2}} \\
\frac{\partial^{2} F_{1}}{\partial n_{1} \partial n_{2}} & \frac{\partial^{2} F_{2}}{\partial n_{1} \partial n_{2}} & \frac{\partial^{2} F_{3}}{\partial n_{1} \partial n_{2}} \\
\frac{\partial^{2} F_{1}}{\partial n_{1} \partial n_{3}} & \frac{\partial^{2} F_{2}}{\partial n_{1} \partial n_{3}} & \frac{\partial^{2} F_{3}}{\partial n_{1} \partial n_{3}} \\
\frac{\partial^{2} F_{1}}{\partial n_{2}{ }^{2}} & \frac{\partial^{2} F_{2}}{\partial n_{2}{ }^{2}} & \frac{\partial^{2} F_{3}}{\partial n_{2}{ }^{2}} \\
\frac{\partial^{2} F_{1}}{\partial n_{2} \partial n_{3}} & \frac{\partial^{2} F_{2}}{\partial n_{2} \partial n_{3}} & \frac{\partial^{2} F_{3}}{\partial n_{2} \partial n_{3}} \\
\frac{\partial^{2} F_{1}}{\partial n_{3}{ }^{2}} & \frac{\partial^{2} F_{2}}{\partial n_{3}{ }^{2}} & \frac{\partial^{2} F_{3}}{\partial n_{3}{ }^{2}}
\end{array}\right)_{\mathbf{n}^{0}(-x,-z)}=\left(\begin{array}{ccc}
+\frac{\partial^{2} F_{1}}{\partial n_{1}{ }^{2}} & -\frac{\partial^{2} F_{2}}{\partial n_{1}{ }^{2}}+\frac{\partial^{2} F_{3}}{\partial n_{1}{ }^{2}} \\
-\frac{\partial^{2} F_{1}}{\partial n_{1} \partial n_{2}} & +\frac{\partial^{2} F_{2}}{\partial n_{1} \partial n_{2}} & -\frac{\partial^{2} F_{3}}{\partial n_{1} \partial n_{2}} \\
+\frac{\partial^{2} F_{1}}{\partial n_{1} \partial n_{3}} & -\frac{\partial^{2} F_{2}}{\partial n_{1} \partial n_{3}} & +\frac{\partial^{2} F_{3}}{\partial n_{1} \partial n_{3}} \\
+\frac{\partial^{2} F_{1}}{\partial n_{2}{ }^{2}} & -\frac{\partial^{2} F_{2}}{\partial n_{2}{ }^{2}}+\frac{\partial^{2} F_{3}}{\partial n_{2}{ }^{2}} \\
-\frac{\partial^{2} F_{1}}{\partial n_{2} \partial n_{3}} & +\frac{\partial^{2} F_{2}}{\partial n_{2} \partial n_{3}}-\frac{\partial^{2} F_{3}}{\partial n_{2} \partial n_{3}} \\
+\frac{\partial^{2} F_{1}}{\partial n_{3}{ }^{2}} & -\frac{\partial^{2} F_{2}}{\partial n_{3}{ }^{2}}+\frac{\partial^{2} F_{3}}{\partial n_{3}{ }^{2}}
\end{array} \mathbf{n}_{\mathbf{n}^{0}(x, z)}\right.
$$


we end with the conclusion that

$$
\left\langle\left. D^{2} \mathbf{F}\right|_{\mathbf{n}^{\circ}}\left(\mathbf{n}^{1}, \mathbf{n}^{1}\right), \frac{\partial \mathbf{n}^{0}}{\partial x}\right\rangle=0 \quad \nu_{\mathrm{e}}\left\langle\mathbf{n}^{1} \wedge \mathcal{E}, \frac{\partial \mathbf{n}^{0}}{\partial x}\right\rangle=0
$$

and this, thanks to the power of symmetry analysis, without any computations! Therefore the right hand side of equation (6) $\left(\mathrm{rhs}^{2}\right)$ is in fact reduced to the single non-vanishing term

$$
-\gamma_{1}\left\langle\frac{\partial \mathbf{n}^{1}}{\partial t_{1}}, \frac{\partial \mathbf{n}^{0}}{\partial x}\right\rangle
$$

This term is proportional to $\mathcal{E}$ and does not depend on $\mathcal{E}^{2}$. The CF1 is then at rest under the application of an ac electric field, as observed experimentally.

In conclusion, we have shown that the Lehmann rotation effect may be at the origin of the drift of the first class cholesteric finger perpendicular to its long-axis. The estimated order of magnitude, as well as the computation of a vanishing velocity under an AC electric field, are both characteristic features in good agreement with the experimental results. However it is worth to remember that $\nu_{\mathrm{e}}$ is just a phenomenological coefficient, derived from symmetry considerations, and that the underlaying microscopic physics, especially the role played by the charge injection mechanism [15], is still not understood. Works in this direction are in progress.

\section{Acknowledgments}

We are very grateful to J. Prost and J.M. Gilli for their fruitful discussions. We also acknowledge the French $\mathbf{D}$ irection des $\mathbf{R}$ echerches et $\mathbf{E}$ tudes $\mathbf{T}$ echnqques, contrat $n^{\circ}=94-2610 A$, for its financial support.

\section{References}

[1] Pomeau Y., Phys. Lett. 34A (1971) 143.

[2] Lehmann O., Ann. Phys. 2 (1900) 649.

[3] Oseen C.W., Trans. Faraday Soc. 29 (1933) 883.

[4] Ericksen J.L., Arch. Ration. Mech. Analysis 4 (1960) 231 and Ericksen J.L., Phys. Fluıds 9 (1966) 1205.

[5] Leslie F.M., Quart. J. Mech. appl. Math. 19 (1966) 357; Leslie F.M., Arch. ration. Mech. Analysis 28 (1968) 265 and Leslie F.M., Proc. R. Soc. A307 (1968) 359.

[6] Lubensky T.C., Mol. Crys. Lrq. Crys. 23 (1973) 99.

[7] Martin P.C., Parodi O. and Pershan P.S. Phys. Rev. A 6 (1972) 2401.

[8] Madhusudana N.V. and Pratibha R., Liq. Cryst. 5 (1989) 1827. Note that the experiments by Eber and Janossi (Mol. Crys. Liq. Crys. Lett. 72 (1982) 233) deal with the measurement of the thermomechanical coupling coefficients and not with electromechanical ones.

[9] Madhusudana N.V., Pratibha R. and Padmini H.P., Mol. Crys. Liq. Crys. 202 (1991) 35.

[10] Padmini H.P. and Madhusudana N.V., Liq. Cryst. 14 (1993) 497.

[11] Ribiere P. and Oswald P., J. Phys. France 51 (1990) 1703.

[12] Lequeux F., Oswald P. and Bechhoefer J., Phys. Rev. A 40 (1989) 3974.

[13] Ribiere P., Oswald P. and Pirkl S., J. Phys. II France 4 (1994) 127.

[14] Gil L., J. Phys. II France 5 (1995) 1819. 
[15] Hinov H.P. and Kukleva E., Mol. Crys. Liq. Crys. 109 (1984) 203.

[16] Gil L., Gilli J.M. and Frisch T., Phys. Rev. E 48 (1993) 4199.

[17] Gilli J.M. and Gil L., Liq. Cryst. 17 (1994) 1.

[18] Felici N.J., J. Phys. Colloq. France, 37 (1976) C1 117.

[19] Gilli J.M. and Kamaye M., Liq. Cryst. 11 (1992) 569.

[20] Chandrasekhar S., Liquid Crystals, Monographs on Physics, (Cambridge University Press, Cambridge, 1980).

[21] Lyapunov A.M., Problème Général de la stabilité du mouvement. Annals of Mathematics Studies $\mathrm{n}^{\circ} 17$, (Princeton University Press, Princeton, NJ, 1947).

[22] Nagaya T., Hikita Y., Orihara H. and Ishibashi Y., J. Phys. Soc. Jpn. 65 (1996) 2713.

[23] Guckenheimer J. and Holmes P., Non Linear Oscillations, Dynamical Systems and Bifurcation of Vector Fields (Springer, Berlin, Heidelberg 1983).

[24] Goldstone J., Nuovo Cimento 19 (1961) 154.

[25] Gilli J. M. and Kamaye M., Liq. Cryst. 11 (1992) 791.

[26] Mitov M. and Sixou P., J. Phys. II France 2 (1992) 1659.

[27] For example, it is possible to numerically check that expressions like $\iint n_{1}^{0} n_{2}^{0} \mathrm{~d} x \mathrm{~d} z$ or $\iint n_{2}^{0} n_{3}^{0} \mathrm{~d} x \mathrm{~d} z$ vanish. 